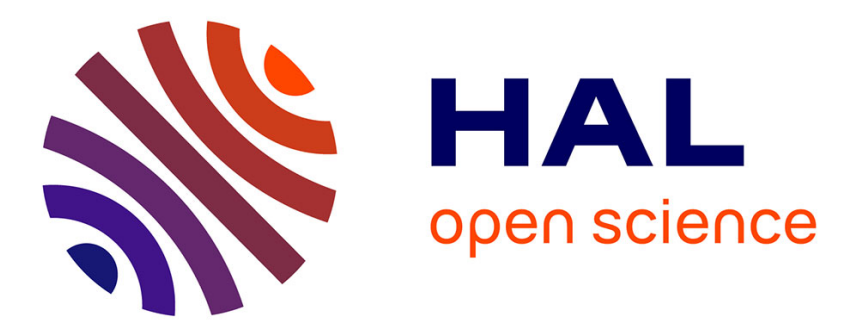

\title{
The ultimate limit to the emission linewidth of single nanocrystals
}

\author{
Mark J. Fernée, Chiara Sinito, Yann Louyer, Philippe Tamarat, Brahim \\ Lounis
}

\section{To cite this version:}

Mark J. Fernée, Chiara Sinito, Yann Louyer, Philippe Tamarat, Brahim Lounis. The ultimate limit to the emission linewidth of single nanocrystals. Nanotechnology, 2013, 24, pp.465703 (1-5). 10.1088/0957-4484/24/46/465703 . hal-00877709

\section{HAL Id: hal-00877709 \\ https://hal-iogs.archives-ouvertes.fr/hal-00877709}

Submitted on 6 Mar 2018

HAL is a multi-disciplinary open access archive for the deposit and dissemination of scientific research documents, whether they are published or not. The documents may come from teaching and research institutions in France or abroad, or from public or private research centers.
L'archive ouverte pluridisciplinaire HAL, est destinée au dépôt et à la diffusion de documents scientifiques de niveau recherche, publiés ou non, émanant des établissements d'enseignement et de recherche français ou étrangers, des laboratoires publics ou privés.

\section{다)(1) $(5$}

Distributed under a Creative Commons Attribution - NonCommerciall 4.0 International 


\title{
The ultimate limit to the emission linewidth of single nanocrystals
}

\author{
Mark J Fernée ${ }^{1,2}$, Chiara Sinito ${ }^{1,2}$, Yann Louyer ${ }^{3}$, Philippe Tamarat ${ }^{1,2}$ \\ and Brahim Lounis ${ }^{1,2}$ \\ ${ }^{1}$ Université Bordeaux, LP2N, F-33405 Talence, France \\ ${ }^{2}$ Institut d'Optique and CNRS, LP2N, F-33405 Talence, France \\ ${ }^{3}$ Université Bordeaux, LOMA, F-33405 Talence, France \\ E-mail: blounis@u-bordeaux1.fr
}

\begin{abstract}
Measurements of the emission linewidth of single nanocrystals are usually limited by spectral diffusion. At cryogenic temperatures, the origin of this instability was revealed to be photo-induced, suggesting that the spectral peak position may be stable in the limit of vanishing optical excitation. Here we test this stability using resonant photoluminescence excitation and find there is persistent spectral broadening, which ultimately limits the emission linewidth in these materials. The spectral broadening is shown to be consistent with spontaneous fluctuations of the local electrostatic field within the disordered environment surrounding the nanocrystal.
\end{abstract}

The optical resonance linewidth of a single quantum emitter provides a direct measurement of decoherence in the system, which is a quantity of interest to certain quantum technological applications and ultimately sets a fundamental limitation for these materials. However, accessing the homogeneous linewidth has proved problematic in semiconductor nanocrystals (NCs) due to the effect of spectral diffusion (SD) [1-3], which is a random fluctuation of the transition energy. Instabilities in the spectral position are discouraging for potential applications in, for example, the coherent control of quantum states $[4,5]$ and coupled systems [6], thus so far there have been few reports using NCs [7]. One note of encouragement is that SD has been attributed to hot-carrier relaxation, which is photo-induced (i.e. requiring the absorption of a photon) [8-10]. If this is the case, then it may be possible that the NC quantum system remains undisturbed between photon absorption events and may be more stable under weak resonant excitation, which avoids the generation and relaxation of hot charge carriers. However, determining if this scenario holds is complicated when the system is interacting with the optical field.

There have been a number of studies that probe the physical origin of SD in NCs [2, 9-16]. The most widely accepted hypothesis is that SD observed in single NCs arises from rearrangements of the local charge environment after excitation. This was primarily established using single NC Stark shift spectroscopy, where it was found that the effects of an externally applied electric field caused shifts of single NC spectra that resembled that observed in SD [11], from which the magnitude of the internal fields were estimated. Further investigations using a more diverse range of spectroscopic tools have established timescales for SD that range from microseconds $[13,14]$ to seconds [10]. In fact, it has been shown that there are two distinct jump rates involved, indicating two distinct processes. The slow rate process has now been well studied and attributed to a photo-induced phenomenon caused by hot-carrier relaxation $[2,10]$. However, although the fast process has been identified, its properties have not been established, apart from the asymptotic millisecond upper bound to the timescale [17]. This fast rate component has ultimately limited the measurement of the spectral linewidth to $1.5 \mathrm{GHz}[13,14,18]$, which is up to two orders of magnitude broader than the lifetime limit. The cause of this residual broadening is still uncertain.

In this paper we use high-resolution resonant photoluminescence excitation (R-PLE) [18] to study SD over a spectral range compatible with the transition frequency fluctuations of the fast-SD process in order to understand the underlying mechanisms and place a lower limit on the single NC 
linewidth. As spectra are acquired by scanning the laser across a spectral line, the line shape itself is a sensitive indicator of the nature of the SD processes, enabling us to discriminate between persistent and photo-induced SD. Finally, we discuss the implications of our findings for potential applications where spectral stability can be critical.

The experiment and samples have been described elsewhere [18-20]. Briefly, commercial water soluble $\mathrm{CdSe} / \mathrm{ZnS}$ core/shell NCs conjugated with streptavidin are diluted to an approximately nanomolar concentration in a $1 \%$ polyvinyl alcohol (PVOH) solution and deposited on a clean glass cover slip via spin coating, resulting in single detectable NCs immobilized in a PVOH matrix. The sample is mounted in a liquid He bath cryostat, where the sample is in contact with a low-pressure He buffer gas that is maintained at $2 \mathrm{~K}$ throughout the experiment. Photoluminescence (PL) is collected using an infinity-corrected $0.95 \mathrm{NA}$ objective mounted inside the cryostat. The resonant excitation experiment (R-PLE) consists of scanning a single-mode $\mathrm{cw}$ dye laser (1 MHz linewidth) across a single NC zero-phonon line (ZPL) and collecting the signal emitted into the optical phonon side band, which is red-shifted approximately $6.5 \mathrm{THz}$ from the ZPL (see diagram, figure 1(a)). A laser scan range of $20 \mathrm{GHz}$ is used in this experiment and a low-pass filter with a sharp edge (see figure 1(a)) is used to remove the scattered laser signal. The filter edge is tuned to the individual $\mathrm{NC}$ by setting the initial scan position slightly red-detuned from the ZPL and then tilting the filter until the laser signal is suppressed. During the experiment a pump irradiance of $50 \mathrm{~W} \mathrm{~cm}^{-2}$ was employed, resulting in an estimated emission rate $\sim 10^{6}$ photons $\mathrm{s}^{-1}$.

The nature in which SD manifests itself in R-PLE scans has been well studied in cryogenic single polyaromatic molecule spectroscopy [21-24]. In particular, photo-induced $\mathrm{SD}$ results in asymmetric spectral line scans similar to that shown in figure 1(b) [21], while persistent (also referred to as spontaneous) SD results in symmetric spectral line scans $[23,24]$. Recently we have shown that the slow-SD process, which is known to be photo-induced, results in asymmetric R-PLE scans [16]. The observation of slow-SD in R-PLE suggests that optical phonons alone can be responsible for the slow-SD process. However, in the case of hot-carrier excitation an additional relaxation channel involving surface states may also be involved $[25,26]$. As the slow-SD process occurs with a rate [10] comparable to the spectral scan rate, it is difficult to disentangle the effects of fast-SD from that of slow-SD. However, since the spectral jump distribution for slow-SD [10] is far greater than that for fast-SD [14], the two processes should be distinguishable in R-PLE spectra.

In order to reduce the effect of slow-SD on the R-PLE scans, we increased our scan rate from $\sim 6$ to $30 \mathrm{GHz} \mathrm{s}^{-1}$. This effectively reduces the dwell time of the excitation laser in resonance with the $\mathrm{NC}$, proportionally lowering the probability of a photo-induced spectral jump. In figure 2(a) we reveal the fast-SD process in high resolution with a series of 22 successive R-PLE scans of a single NC spectral line. The frequency range of the scans is comparable to the spectral broadening previously reported for the fast-SD process [14].

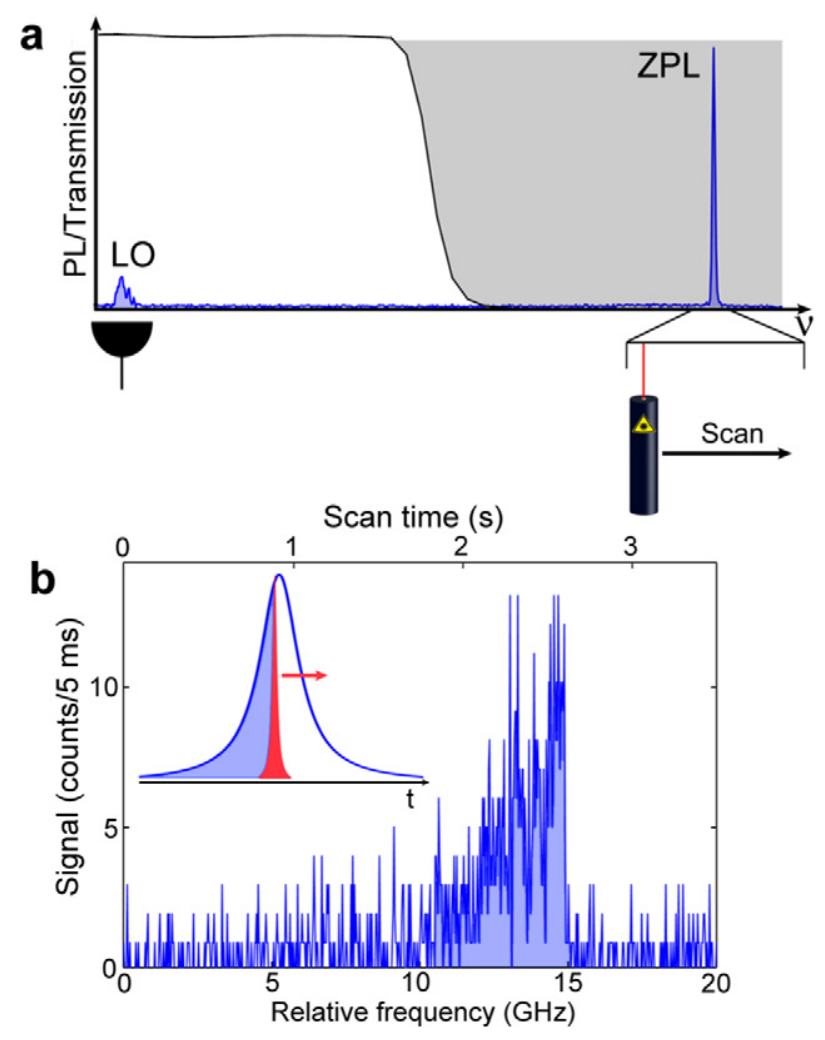

Figure 1. (a) Schematic of the R-PLE scanning process where the single-mode laser is scanned across the NC spectral line (ZPL) and the signal is detected using the LO phonon replica. A low-pass filter (curve shown) is used to remove the scattered laser (shaded area) from the detected signal. (b) A $20 \mathrm{GHz}$ R-PLE scan of a single NC at $2 \mathrm{~K}$ revealing an asymmetric line shape due to a spectral jump occurring midway through the scan (inset illustrates the scan progress as a shaded region).

All the individual scans are quite symmetric, exhibiting both smoothly rising and falling edges. The fast-SD process is revealed by fitting each individual spectrum with a Gaussian function using a nonlinear least squares algorithm in order to determine the peak position and estimate the spectral fluctuation width. Here we see that the peak position wanders over a range of approximately $3 \mathrm{GHz}$ and the width varies over a similar range, indicating that SD strongly affects the individual scans.

In order to estimate the underlying homogeneous linewidth of the NC we sum the scans, which we plot in figure 2(b). We find a good fit to the summed signal using a Voigt profile with a Gaussian contribution of $4.4 \mathrm{GHz}$ full width at half maximum (FWHM), and a Lorentzian contribution of $1.5 \mathrm{GHz}$ FWHM. The Lorentzian FWHM represents an upper estimate to the homogeneous linewidth (as larger Lorentzian widths noticeably deviate with the data in the wings of the distribution). In addition we see that there is a second spectral position, which can be attributed to SD induced by rapid flipping of a bistable environmental charge distribution, as observed with single molecules close to an asymmetric two-level-system in a disordered matrix [21-24, 29]. In general, we have applied the R-PLE technique to tens 

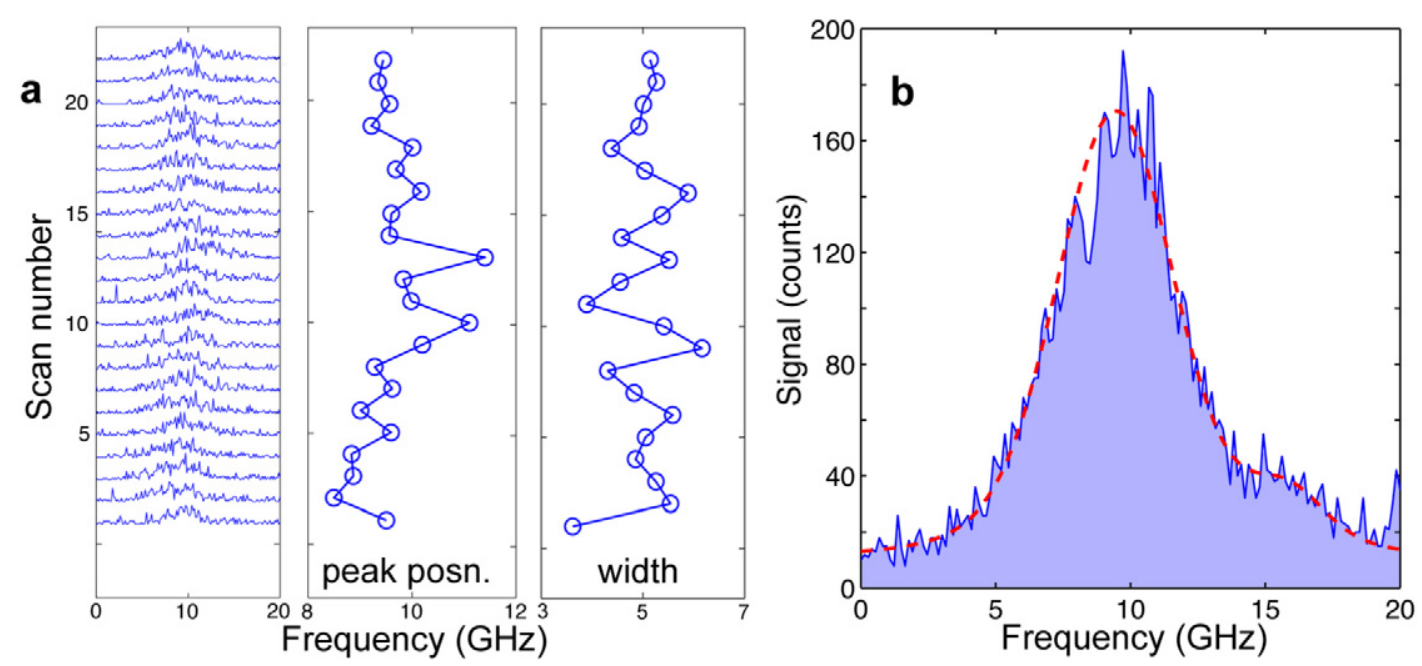

Figure 2. (a) A series of 22 successive high-resolution R-PLE scans of a single NC spectral line revealing SD in the detected signal. The peak positions and estimated fluctuation width are obtained by least squares fitting of a Gaussian. (b) The sum of 22 single scans depicted in (a) with an associated Voigt curve (red dashed) corresponding to a 1.5 GHz FWHM Lorentzian component and a 4.4 GHz FWHM Gaussian component (as well as a $2.6 \mathrm{GHz}$ Gaussian for the side peak).

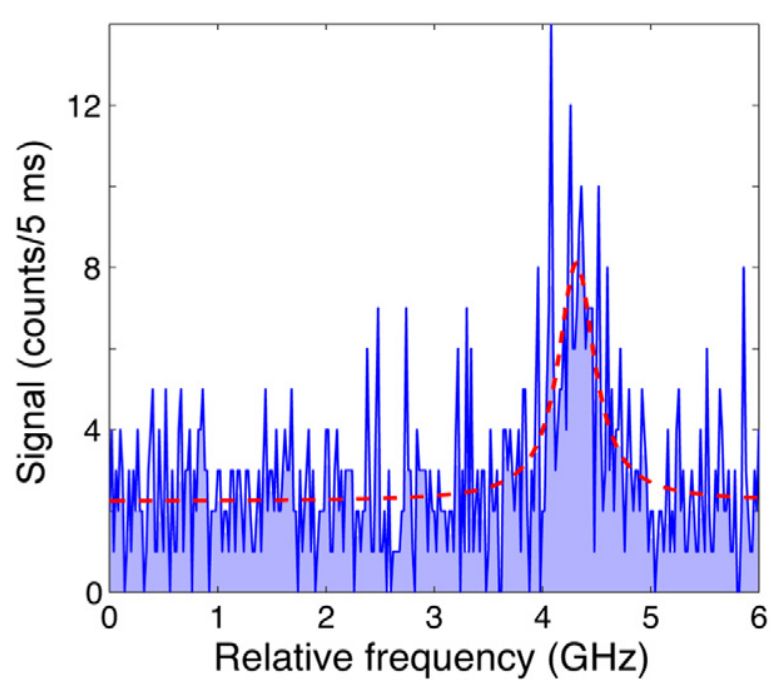

Figure 3. The narrowest obtained R-PLE scan fit with a $400 \mathrm{MHz}$ FWHM Lorentzian curve.

of single NCs and find symmetric R-PLE scans of single $\mathrm{NC}$ lines as narrow as approximately $1 \mathrm{GHz}$, with the narrowest single scan of $400 \mathrm{MHz}$ shown in figure 3. Such a scan is compatible with the homogeneous broadening limit established with the data in figure 2 and provides an indication of the ultimate limit to the spectral width in these materials.

The linewidths we obtain represent an experimental timescale close to the $1 \mathrm{~s}$ range, which is significantly slower than the millisecond timescale reported for asymptotic broadening of the spectral line due to fast-SD [14], indicating that these are in fact fast-SD-limited linewidths. Nevertheless, these represent the narrowest linewidths yet reported. Most importantly, the generally symmetric lineshapes indicate that the fast-SD process occurs independently of the pump laser, thus revealing a fundamental limit to the spectral stability of these materials.
We note our R-PLE studies are based on NCs that have been carefully selected for spectral stability using the criterion of NCs that exhibit resolution-limited photoluminescence lines [27]. This selection is necessary in order to locate the spectral line with the R-PLE scan due to the narrow scan range of the laser. Such NCs are likely to be those near the quadratic Stark effect regime [11], making them less sensitive to external charge fluctuations. Furthermore, we operate at the lowest operable temperature of $2 \mathrm{~K}$, where acoustic phonon populations are suppressed in NCs [28]. Nevertheless, of this ultra-stable fraction (comprising tens of $\mathrm{NCs}$ ), we find that all are broadened far beyond their lifetime limit [29]. The likely source of this broadening is charge noise in the surrounding matrix. As the exciton states in NCs are highly polarizable [11], they are capable of detecting environmental charge noise and it is known that such charge noise persists in random polymer matrices to the lowest experimental temperatures [24, 30, 31].

We estimate the spectral jump magnitude for fast-SD to be in the $1 \mathrm{GHz}$ range, using the SD data in figure 2 . This is more than an order of magnitude smaller than the spectral jumps associated with the slow-SD process [10]. In order to try to understand the origin of this fast-SD, we recall that the slow-SD process is generally associated with charge displacements on the surface of the $\mathrm{NC}[2,9,10,16]$, which enables us to make a relative comparison of the two processes as follows: we associate the instantaneous spectral position with the presence of local fields $\xi_{1}$ which undergo fluctuations $\Delta \xi_{1}$ associated with SD. Then the shift in the emission energy, $\Delta E$, due to fast-SD is given by $\Delta E=$ $2 \alpha \xi_{1} \Delta \xi_{1}$, where $\alpha$ is the polarizability of the NC. We can approximate $\Delta \xi_{1}$ as a far-field dipolar field due to some charge displacement according to $\Delta \xi_{1}=p / 4 \pi \varepsilon_{0} \varepsilon_{\mathrm{r}} r^{3}$, where $p$ is the associated dipole and $r$ is the distance to the center of the NC. Using this simple picture, the difference in magnitude between the fast-SD and slow-SD processes either represents proportionally smaller $(<0.1 \times)$ charge displacements or 
similar charge displacements occurring more than twice the distance from the center of the $\mathrm{NC}$. We can estimate the dipole change in the matrix using known values from the literature. The dipole change associated with the flip of a two-level-system in a polymethylmethacrylate (PMMA) polymer is $0.4 \mathrm{D}$ [24], while that associated with similar processes in undoped silicon is $0.6 \mathrm{D}$ [24]. We use the lower value for PMMA, a relative permittivity of $\varepsilon_{\mathrm{r}}=5$ and a radius of $10 \mathrm{~nm}$, giving $\Delta \xi_{1}=25 \mathrm{~V} \mathrm{~cm}^{-1}$. Comparing with the Stark shift data of Empedocles and Bawendi [11] using a local field of $90 \mathrm{kV} \mathrm{cm}{ }^{-1}$ and polarizability of $3.2 \times 10^{5} \AA^{3}$ (corresponding to values reported for the largest $\mathrm{NCs}$ ), we estimate that such dipole changes would give rise to energy shifts in the $\mathrm{GHz}$ range. This estimate indicates that NCs should be sensitive to relatively small charge fluctuations in their surrounding environment, which supports our hypothesis that charge fluctuations in the disordered polymer environment are the origin for the fast-SD. Thus fast-SD may pose a fundamental limit to the application of NCs in quantum technologies, where unwanted environmental influences must be minimized.

The fast-SD process in NCs has been studied using the high-resolution R-PLE technique and found not to be photo-induced, but instead is attributed to charge fluctuations within the surrounding organic matrix of the NC. The persistence of this form of $\mathrm{SD}$ at $2 \mathrm{~K}$ is similar to processes observed in random organic matrices [24, 30, 31] and will therefore serve as a fundamental limitation to the adoption of these materials in applications requiring a stable and narrow linewidth. However, the $<10 \mathrm{GHz}$ stability of these NCs may be good enough for applications in a range of strongly coupled systems [7, 32, 33] where coupling energies $>10 \mathrm{GHz}$ are predicted. Ultimately, our results suggest that strategies aimed towards mitigating SD should be directed towards increasing the distance from the NC core to the organic surface layer, as well as decreasing the polarizability of the $\mathrm{NC}$, which can be accomplished using small cores that strongly confine both charge carriers.

\section{References}

[1] Empedocles S A, Norris D J and Bawendi M G 1996 Photoluminescence spectroscopy of single CdSe nanocrystallite quantum dots Phys. Rev. Lett. 77 3873-6

[2] Empedocles S A and Bawendi M G 1999 Influence of spectral diffusion on the line shapes of single CdSe nanocrystallite quantum dots J. Phys. Chem. B 103 1826-30

[3] Blanton S A, Hines M A and Guyot-Sionnest P 1996 Photoluminescence wandering in single CdSe nanocrystals Appl. Phys. Lett. 69 3905-7

[4] Press D, Ladd T D, Zhang B Y and Yamamoto Y 2008 Complete quantum control of a single quantum dot spin using ultrafast optical pulses Nature 456 218-21

[5] Xu X, Sun B, Berman P R, Steel D G, Bracker A S, Gammon D and Sham L J 2008 Coherent population trapping of an electron spin in a single negatively charged quantum dot Nature Phys. 4 692-5

[6] Hennessy K, Badolato A, Winger M, Gerace D, Atature M, Gulde S, Falt S, Hu E L and Imamoglu A 2007 Quantum nature of a strongly coupled single quantum dot-cavity system Nature $\mathbf{4 4 5}$ 896-9
[7] Le Thomas N, Woggon U, Schops O, Artemyev M V, Kazes M and Banin U 2006 Cavity QED with semiconductor nanocrystals Nano Lett. 6 557-61

[8] Empedocles S A, Neuhauser R, Shimizu K and Bawendi M G 1999 Photoluminescence from single semiconductor nanostructures $A d v$. Mater. 11 1243-56

[9] Muller J, Lupton J M, Rogach A L, Feldmann J, Talapin D V and Weller H 2005 Monitoring surface charge migration in the spectral dynamics of single $\mathrm{CdSe} / \mathrm{CdS}$ nanodot/nanorod heterostructures Phys. Rev. B 72205339

[10] Fernée M J, Littleton B, Plakhotnik T, Rubinsztein-Dunlop H, Gómez D E and Mulvaney P 2010 Charge hopping revealed by jitter correlations in the photoluminescence spectra of single CdSe nanocrystals Phys. Rev. B 81155307

[11] Empedocles S A and Bawendi M G 1997 Quantum-confined Stark effect in single CdSe nanocrystallite quantum dots Science 278 2114-7

[12] Neuhauser R G, Shimizu K T, Woo W K, Empedocles S A and Bawendi M G 2000 Correlation between fluorescence intermittency and spectral diffusion in single semiconductor quantum dots Phys. Rev. Lett. 85 3301-4

[13] Palinginis P, Tavenner S, Lonergan M and Wang H 2003 Spectral hole burning and zero phonon linewidth in semiconductor nanocrystals Phys. Rev. B 67 201307(R)

[14] Coolen L, Brokmann X, Spinicelli P and Hermier J P 2008 Emission characterization of a single $\mathrm{CdSe}-\mathrm{ZnS}$ nanocrystal with high temporal and spectral resolution by photon-correlation Fourier spectroscopy Phys. Rev. Lett. 100027403

[15] Plakhotnik T, Fernée M J, Littleton B, Rubinsztein-Dunlop H, Potzner C and Mulvaney P 2010 Anomalous power laws of spectral diffusion in quantum dots: a connection to luminescence intermittency Phys. Rev. Lett. 105167402

[16] Fernée M J, Plakhotnik T, Louyer Y, Littleton B N, Potzner C, Tamarat P, Mulvaney P and Lounis B 2012 Spontaneous spectral diffusion in CdSe quantum dots J. Phys. Chem. Lett. 3 1716-20

[17] Littleton B N, Fernée M J, Gomez D E, Mulvaney P and Rubinsztein-Dunlop H 2009 High-resolution line width measurement of single CdSe nanocrystals at long time scales J. Phys. Chem. C 113 5345-8

[18] Biadala L, Louyer Y, Tamarat P and Lounis B 2009 Direct observation of the two lowest exciton zero-phonon lines in single CdSe/ZnS nanocrystals Phys. Rev. Lett. 103037404

[19] Biadala L, Louyer Y, Tamarat P and Lounis B 2010 Band-edge exciton fine structure of single $\mathrm{CdSe} / \mathrm{ZnS}$ nanocrystals in external magnetic fields Phys. Rev. Lett. 105157402

[20] Louyer Y, Biadala L, Trebbia J B, Fernée M J, Tamarat P and Lounis B 2011 Efficient biexciton emission in elongated CdSe/ZnS nanocrystals Nano Lett. 11 4370-5

[21] Orrit M and Bernard J 1990 Single pentacene molecules detected by fluorescence excitation in a P-terphenyl crystal Phys. Rev. Lett. 65 2716-9

[22] Basché T and Moerner W E 1992 Optical modification of a single impurity molecule in a solid Nature $355335-7$

[23] Reilley P D and Skinner J L 1993 Spectral diffusion of single molecule fluorescence: a probe of low-frequency localized excitations in disordered crystals Phys. Rev. Lett. 71 4257-60

[24] Donley E A, Bach H, Wild U P and Plakhotnik T 1999 Coupling strength distributions for dynamic interactions experienced by probe molecules in a polymer host J. Phys. Chem. A 103 2282-9

[25] Cooney R R, Sewall S L, Anderson K E H, Dias E A and Kambhampati P 2007 Breaking the phonon bottleneck for holes in semiconductor quantum dots Phys. Rev. Lett. 98177403

[26] Kambhampati P 2010 Unraveling the structure and dynamics of excitons in semiconductor quantum dots Acc. Chem. Res. 44 1-13 
[27] Fernée M J, Tamarat P and Lounis B 2013 Cryogenic single-nanocrystal spectroscopy: reading the spectral fingerprint of individual CdSe quantum dots J. Phys. Chem. Lett. 4 609-18

[28] Fernée M J, Sinito C, Louyer Y, Potzner C, Nguyen T-L, Mulvaney P, Tamarat P and Lounis B 2012 Magneto-optical properties of trions in non-blinking charged nanocrystals reveal an acoustic phonon bottleneck Nature Commun. 31287

[29] Fernée M J, Louyer Y, Tamarat P and Lounis B 2012 Comment on 'Spin-flip limited exciton dephasing in $\mathrm{CdSe} / \mathrm{ZnS}$ colloidal quantum dots' Phys. Rev. Lett. 109229701
[30] Tittel J, Kettner R, Basché T, Brauchle C, Quante H and Mullen K 1995 Spectral diffusion in an amorphous polymer probed by single molecule spectroscopy J. Lumin. 64 1-11

[31] Boiron A M, Tamarat P, Lounis B, Brown R and Orrit M 1999 Are the spectral trails of single molecules consistent with the standard two-level system model of glasses at low temperatures? Chem. Phys. 247 119-32

[32] Mandal S, Serey X and Erickson D 2010 Nanomanipulation using silicon photonic crystal resonators Nano Lett. 10 99-104

[33] Waks E and Sridharan D 2010 Cavity QED treatment of interactions between a metal nanoparticle and a dipole emitter Phys. Rev. A 82043845 